\title{
Intelligent Identification of Coal Structure for the Control of Heat-Induced Gas Outburst and Energy-Efficient Mining
}

\author{
Tao Li ${ }^{1,4}$, Banghua Yao ${ }^{2,3,5^{*}}$, Zhihui Wen ${ }^{2,3,5}$, Dengke Wang ${ }^{2,3,5}$, Hongtu Zhang ${ }^{2,3,5}$ \\ ${ }^{1}$ School of Emergency Management and Safety Engineering, China University of Mining and Technology-Beijing, Beijing \\ 100083, China \\ ${ }^{2}$ School of Safety Science and Engineering, Henan Polytechnic University, Jiaozuo 454000, China \\ ${ }^{3}$ State Key Laboratory Cultivation Base for Gas Geology and Gas Control (Henan Polytechnic University), Jiaozuo 454000, \\ China \\ ${ }^{4}$ School of Electrical Engineering \& Automation, Henan Polytechnic University, Jiaozuo 454000, China \\ ${ }^{5}$ State Collaborative Innovation Center of Coal Work Safety and Clean-Efficiency Utilization, Jiaozuo 454000, China
}

Corresponding Author Email: yaobanghua@126.com

https://doi.org/10.18280/ijht.380409

Received: 13 July 2020

Accepted: 28 September 2020

\section{Keywords:}

type of coal structure, heat-induced gas outburst, intelligent identification, energyefficient mining

\begin{abstract}
Coal structure could be roughly divided into four types. Among them, the two kinds of tectonic coal face a high risk of heat-induced gas outburst, which arises from the unfavorable temperature conditions in the coal structure. However, there is not yet an efficient way to identify the type of coal structure. The adjacent types of coal structures are often misjudged. The lack of an efficient identification method hinders the prevention of heat-induced gas outburst, making it difficult to realize energy-efficient and safe mining. To solve the problem, this paper first theoretically analyzes the ultrasonic properties of different types of coals, and applies backpropagation neural network (BPNN) to build up an intelligent identification model for the type of coal structure. Specifically, the characteristic parameters of ultrasonic signal were taken as the basis for judging the type of coal structure, the identification algorithm of BPNN was adopted to accurately identify the structure type of coal, and then the heat-induced gas outburst risk of the coal was evaluated preliminarily. Experimental results show that the proposed model could accurately identify the type of coal structure, and even differentiate between adjacent types of coals. The research results provide a reference for effective prevention of heat-induced gas outburst, and realization of energy-efficient and safe mining.
\end{abstract}

\section{INTRODUCTION}

Coal is a valuable resource to economic development. However, heat-induced gas outburst occurs occasionally in coalmining, bringing serious property losses and casualties. Heat-induced gas outburst refers to the gas outburst caused by the unfavorable temperature conditions in the coal structure. To safeguard production and realize energy-efficient mining, it is important to prevent heat-induced gas outburst in a scientific and effective manner.

Heat-induced gas outburst may arise from complex reasons. From different angles, many scholars have expounded the mechanism of heat-induced gas outburst, according to the main factors of this disaster $[1,2]$. Despite holding different views, they agree that heat-induced gas outburst is closely associated with tectonic coal. Therefore, the detection and identification of tectonic coal is of great significance to the prevention of heat-induced gas outburst and realization of energy-efficient mining.

Studies $[1,2]$ have shown that coal could be divided into four types: primary structure coal (type I), fractured coal (type II), pulverized coal (type III), and mylonitic coal (type IV). The four types of coal differ in the level of heat-induced gas outburst risk. Among them, type I coal has no risk of heatinduced gas outburst, type II coal has low to moderate risk, while types III and IV coals have high risk. Conventionally, types I and II coals are referred to as non-outburst coals, while types III and IV coals are called outburst coals, a.k.a., tectonic coal.

Currently, tectonic coal is mainly detected on a regional basis [3-6]. But there is a severe lack of detection of tectonic coal in the small area before the workface, not to mention the accurate discrimination between different types of coal. In this case, it is easy to misjudge the coal type, which negatively affects the prevention of heat-induced gas outburst.

Scholars like Shumskii et al. [7-9] pioneered the application of ultrasonic technology to coal structure research, and obtained fruitful results on the ultrasonic features of disturbed coal, attenuation of acoustic wave in coal, and the ultrasonic estimation of coal structure and fissures. Ultrasonic testing has also been introduced to non-destructive testing [10-15], and achieved success in detecting internal damage of concrete and inspecting human organs [16-19].

In recent years, neural network has attracted much attention from the academia. Song and Miao [20] implemented backpropagation neural network (BPNN) in damage prediction and risk assessment, and demonstrated the good effect of BPNN in predictive analysis and intelligent discrimination [21, 22]. By improving the backpropagation algorithm, Chang et al. [23-25] further enhanced the accuracy 
of BPNN in self-learning, prediction, and decision-making.

This paper carries out theoretical and experimental research into the ultrasonic properties of coals with different types of structure, and clarifies the relationship between the main parameters of ultrasonic signal and the types of coal structure. On this basis, a BPNN-based intelligent identification model was established for the type of coal structure, which intelligently distinguishes between different types of coal, although adjacent types of coal structures have partly overlapped identification parameters. The research results provide a strong support to the prevention of heat-induced gas outburst and energy-efficient mining.

This paper mainly includes three parts: Firstly, the ultrasonic properties of each type of coal structure were theoretically summarized, the relationship between the main parameters of ultrasonic signal and the types of coal structure was clarified, and the primary parameters of the identification model were determined; Next, BPNN technology was applied to build up an intelligent identification model for the type of coal structure; Finally, the ultrasonic properties of different types of coals were investigated through experiments, the experimental results were adopted for BPNN learning and training, and the accuracy and reliability of our model were verified.

\section{ULTRASONIC PROPERTIES OF COAL}

The propagation of ultrasound in the medium depends directly on the structural features of the medium. Different types of coal have significant differences in internal structure. From type I to type IV, the degree of damage gradually deepens, the porosity continues to increase, and the density and strength decrease significantly. These structural differences bring marked variations in ultrasonic properties. Therefore, it is technically feasible to identify the type of coal structure through ultrasonic detection.

\subsection{Ultrasonic velocity}

Ultrasonic velocity is an important physical parameter of ultrasound. It refers to the velocity at which the ultrasound propagates in elastic media. There are two kinds of ultrasonic velocity: transverse velocity and longitudinal velocity. The latter is more frequently used in practice. According to the wave equation of ultrasonic wave in coal, the longitudinal ultrasonic velocity can be expressed as:

$$
V_{p}=\sqrt{\frac{\delta+2 \mu}{\rho}}
$$

where, $\delta=\frac{E \sigma}{(1+\sigma)(1-\sigma)}$ is the elastic coefficient, a.k.a. Lamé coefficient, of the medium; $\mu=\frac{E}{2(1+\sigma)}$ is the shear coefficient of the medium; $\mathrm{E}$ is the elastic modulus, a.k.a. Young's modulus, of the medium; $\sigma$ is the Poisson's ratio of the medium. These elastic parameters have close correlations with the properties of the coal. There is the following relationship between $\mathrm{E}, \mu$ and $\sigma$ :

$$
\frac{E}{2 \mu}=1+\sigma
$$

Sorting (1) and (2), the longitudinal ultrasonic velocity can be rewritten as:

$$
V_{p}=\sqrt{\frac{E(1-\sigma)}{\rho(1+\sigma)(1-2 \sigma)}}
$$

Formula (3) shows that the ultrasonic velocity is closely related to the elastic modulus, Poisson's ratio, and the density of the medium, all of which are key parameters to characterize the internal structure of coal.

The medium density has a greater influence on the elastic modulus than on the ultrasonic velocity. Therefore, the greater the density of coal, the faster the ultrasonic velocity. The two factors have an approximately proportional relationship [19].

Different types of coal have sharp differences in elastic modulus and density. Thus, the same ultrasound must propagate at different velocities in different coal structures. The velocity difference is particularly prominent between outburst coals and non-outburst coals. As a result, the ultrasonic velocity in coal can serve as a primary criterion for identifying the type of coal structure.

\subsection{Ultrasonic attenuation coefficient}

The attenuation of ultrasound in the medium is an important aspect of ultrasonic research. The degree of attenuation has a close relationship with the internal structure and performance of the medium material [18]. Hence, the study on ultrasonic attenuation in coal helps to detect the internal structure and performance of the medium.

By the cause of the weakening of sound intensity, sound wave attenuation can be divided into three categories: absorption attenuation, scattering attenuation, and diffusion attenuation. Only absorption attenuation $a_{r}$, and scattering attenuation $a_{s}$ are associated with the structural features of the medium. Thus, diffusion attenuation is generally not considered in the discussion on the relationship between sound wave and coal properties. The total attenuation coefficient for ultrasound in coal can be expressed as:

$$
a=a_{r}+a_{s}=a f+b f^{2}+c f^{4}
$$

where, $f$ is the frequency of ultrasound; $a, b$, and $c$ are the absorption and scattering constants of the medium. Formula (4) shows a significant positive correlation between ultrasonic attenuation coefficient and ultrasonic frequency: the higher the frequency, the larger the attenuation coefficient.

As the ultrasound passes through the coal, the sound pressure attenuates exponentially with the growth in distance $x$ :

$$
p=p_{0} \cdot e^{-a x}
$$

where, $\mathrm{p}_{0}$ is the sound pressure at $x=0$, i.e., the sound source; $\mathrm{p}$ is the sound pressure at the position with a distance of $x$ to the sound source; $e=2.71828$ is the base of the natural logarithm. Formula (5) shows the important role of attenuation coefficient in ultrasound propagation. Without considering diffusion, the attenuation coefficient solely hinges on coal properties. The value of $a$ represents how much coal affects the ultrasonic attenuation. Taking natural logarithm on both sides of formula (5): 


$$
a=\frac{1}{x} \ln \frac{p_{0}}{p}
$$

Since the sound pressure is directly proportional to the amplitude of the vibration displacement of the medium particle, $p$ and $p_{0}$ in formula (6) can be substituted by the corresponding vibration displacements $A$ and $A_{0}$. Then, formula (6) can be transformed into:

$$
a=\frac{1}{x} \ln \frac{A_{0}}{A}
$$

In a word, the attenuation coefficient $a$ is closely related to the internal structure of coal, and different structures of coal are bound to have varied $a$ values [17]. Therefore, attenuation coefficient could serve as a key parameter in the identification of the type of coal structure. In addition, formula (4) reflects the close association between attenuation coefficient and ultrasonic frequency. This means the impact of ultrasonic frequency must be considered while applying the attenuation coefficient.

\section{BPNN-BASED IDENTIFICATION MODEL}

\subsection{Basic model}

Through the above analysis on ultrasonic properties of coal, it was confirmed that the coal structure has much to do with the velocity and attenuation coefficient of ultrasound in coal. Owing to their associations with coal structure, the two parameters provide the basis for identifying the type of coal structure. However, the parameter values are partly overlapped for adjacent types of coal structures, sowing the seed for misjudgment.

Thanks to its complex algorithm and powerful self-learning ability, artificial neural network (ANN) is very suitable for the classification of complex objects. Therefore, this paper attempts to establish an accurate identification model for the type of coal structure based on the ANN.

BPNN is the most mature and popular feedforward network. The network adopts the backpropagation algorithm: in most cases, the information flows forward layer by layer; during weight learning, however, the error between the expected and actual outputs is propagated backward to modify the weight of each layer [21].

BPNN is known for its good robustness and fault tolerance. For a large network, the overall result of BPNN will not be affected if some nodes or edges fail. In addition, the network could continuously improve itself through self-learning. Considering these advantages, this paper constructs a BPNNbased identification model (Figure 1) for the type of coal structure, in view of the ultrasonic properties of coal.

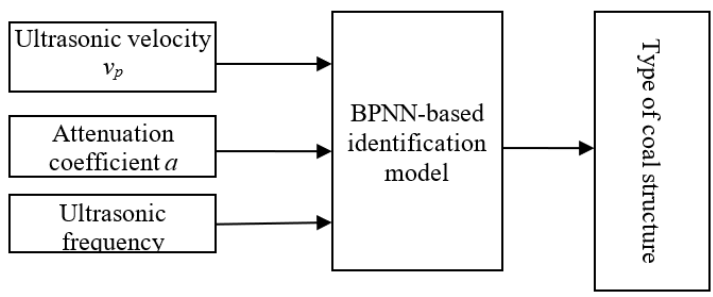

Figure 1. The structure of the BPNN-based identification model
BPNN typically contains an input layer, an output layer, and a hidden layer. As shown in Figure 1, the BPNN-based model takes the ultrasonic parameters that are closely associated with coal structure as the evaluation indies, including the velocity and attenuation coefficient of ultrasound in coal, as well as the auxiliary index of ultrasonic frequency. Meanwhile, the model outputs the type of coal structure. Hence, the input layer has three nodes, and the output layer has one node. The number of nodes in the hidden layer was preliminarily selected by $j=$ $\sqrt{m+n}+l$, where $\mathrm{m}$ and $\mathrm{n}$ are the number of input layer nodes and output layer nodes, respectively; $l$ is a constant in $[1,10]$. The preliminary number was adjusted gradually through repeated tests. Finally, it was determined that the ideal result could be achieved, when the hidden layer has 10 nodes.

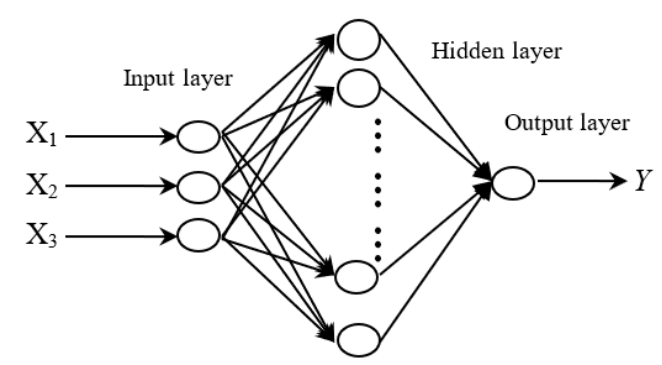

Figure 2. The topology of the BPNN-based identification model

The topology of the BPNN-based identification model is illustrated in Figure 2, where $X_{i}(i=1,2,3)$ is the input $\left(X_{1}, X_{2}\right.$, and $X_{3}$ are ultrasonic velocity, attenuation coefficient, and ultrasonic frequency, respectively); $\mathrm{Y}$ is the output (the ideal values of $Y$ is $1,2,3$, or 4, which represent the four types of coal structures; if $Y=1$ or 2 , the coal has a low risk of heatinduced gas outburst; if $Y=3$ or 4 , the coal has a high risk of heat-induced gas outburst).

\subsection{Backpropagation algorithm and improvement}

BPNN is a feedforward network supporting mapping transform. The basic idea of backpropagation algorithm is as follows: the error between output and fact during network learning are attributed to the faults of the connecting weights and thresholds between the nodes of different layers, the reference errors of each connected node are calculated by apportioning the output error reversely towards the input layer, and the connection weights are adjusted to make the network adapt to the mapping requirements. The connection weights and thresholds are corrected by the generalized delta rule [22]. That is, the weight coefficients are corrected by:

$$
\begin{gathered}
W_{k+1}=W_{k}+\eta \cdot\left(b_{k}-W_{k}^{T} \cdot X_{k}\right) \cdot X_{k} \text { or } \Delta W_{k} \\
=\eta \cdot \delta_{k} \cdot X_{k}
\end{gathered}
$$

where, $\delta_{k}=\left(b_{k}-W_{k}^{T} \cdot X_{k}\right)$ is the difference between the expected output $\mathrm{b}$ and the actual output; $\eta$ is the learning factor. Under certain conditions, with the growing number of iterations $\mathrm{k}, \delta_{k}$ gradually approaches zero to ensure network convergence.

Despite its various advantages, backpropagation algorithm faces several defects. The most prominent defect is the slow convergence, especially in complex networks. The network converges slowly because the error is a complex nonlinear function of time. In essence, backpropagation algorithm is a 
simple steepest descent method. Each weight is adjusted based on the partial derivative of the error to the weight, that is, in the direction of the smallest error rate. As the network is about to converge, the convergence slows down due to $F^{\prime}(x)=0$. Meanwhile, the weight increment $W_{i j}=\eta \delta_{i} X_{i}$, where the coefficients correct the weight to different degrees, while the $\eta$ value remains the same. In this case, some coefficients might be overcorrected. Thus, the network only converges at a very small $\eta$.

In backpropagation algorithm, the key difficulty is the section of step length. If the step length is too long, the learning process will oscillate, failing to obtain convergent network weights; if the step length is too short, the learning will slow down.

To overcome these defects, the backpropagation algorithm needs to be improved. Most of the improvements are based on linear search or Newton's iteration [24, 25]. These methods could reduce the learning cycles and shorten the learning time. But the optimal step length should change significantly over time, and the change should be related to the steepness of the error surface and the curvature of the energy surface. Let $\theta$ be an included angle. Then, the included angle between the n-th and $n-1$-th cycles can be expressed as:

$$
\cos \theta=\frac{\sum_{c} d O_{i} d l_{i}}{\left(\sum_{c} d^{2} O_{i}\right)\left(\sum_{c} d^{2} l_{i}\right)}
$$

where, $d Q_{i}=\left.\frac{\partial E}{\partial \omega_{i}}\right|_{n=n-1} ; d l_{i}=\left.\frac{\partial E}{\partial \omega_{i}}\right|_{n=n} ; c$ is the set of all weights in the network.

The step length should be controlled under the following principles:

(1) For the area where the error surface is relatively flat, the search path is relatively straight and $\cos \theta$ is greater than zero; in this case, the step length should be increased.

(2) After encountering a valley $(\cos \theta<0)$, it is possible to prevent oscillations, but impossible to judge whether the valley is the local minimum or the desired global minimum; in this case, the search speed should be slowed down.

Under the above principles, $\eta_{t}$ was set to $\exp (u \cos \theta) \eta_{t-1}$. Experimental results show that the best effect was achieved at $u=0.1$.

Moreover, since any search method is risky, the minimum and maximum of $\eta$ were determined through experiments. To prevent the oscillation caused by excessive $\eta$, the momentum factor $\alpha$ can be added to the weight coefficient correction formula:

$$
W_{i j}(t+1)=W_{i j}(t)+\eta_{t} \cdot \frac{\partial E}{\partial W_{i j}}+\alpha \Delta \omega_{i j}(t)
$$

$$
\theta_{i j}(t+1)=\theta_{i j}(t)-\eta_{t} \cdot \frac{\partial E}{\partial \theta_{i j}}+\alpha \Delta \theta_{i j}(t)
$$

In this way, the previous weight change was introduced to the weight coefficient. The role of the momentum is equivalent to filtering the higher-order components of the error surface in the weight space. If there is a long gentle valley with steep walls in the weight space, oscillations will occur in the valley. The momentum factor helps to increase the step length, thus speeding up the learning. So far, the authors have completed the construction of the BPNN-based identification model for the type of coal structure.

\section{EXPERIMENTS}

\subsection{Ultrasonic velocity experiments}

This subsection studies the ultrasonic velocities in different types of coal structure through experiments, aiming to clarify the relationship between the velocity and each type of coal structure. As shown in Figure 3, the ultrasonic velocity experiments were conducted by the thru-beam method. During the experiments, the ultrasonic transmitter and receiver were deployed at the two ends of the coal sample, respectively. Then, the computer-controlled power supply generated an oscillating current that matches the transmitter to excite the ultrasonic signal. After the ultrasonic wave passed through the coal sample, the ultrasonic velocity was calculated by dividing the sample length by the propagation time.

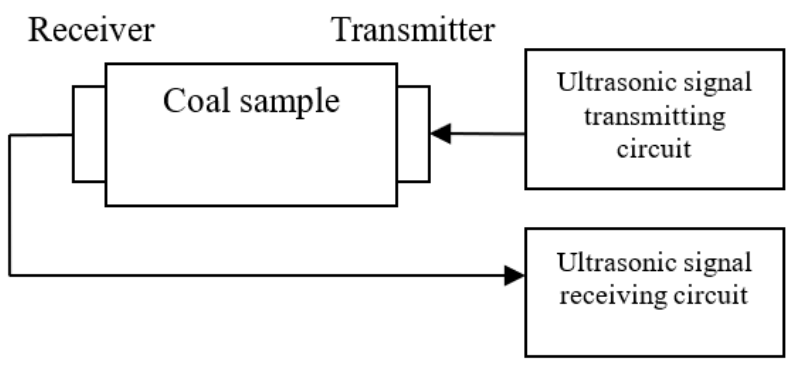

Figure 3. The system structure for ultrasonic velocity experiments

According to the experimental contents and principles, coal samples were collected from different mines for ultrasonic velocity experiments. The ultrasonic velocity of each sample group was determined as the average of repeated measurements, and round to the nearest integer. Some of the experimental data are listed in Table 1.

Table 1. The ultrasonic velocities of some coal samples

\begin{tabular}{ccccc}
\hline Sample source & $\begin{array}{c}\text { Type of coal } \\
\text { structure }\end{array}$ & $\begin{array}{c}\text { Sample length } \\
\mathbf{L}(\mathbf{c m})\end{array}$ & $\begin{array}{c}\text { Interval between transmission } \\
\text { and reception times t }(\boldsymbol{\mu s})\end{array}$ & $\begin{array}{c}\text { Ultrasonic velocity V } \\
(\mathbf{m} / \mathbf{s})\end{array}$ \\
\hline Jiaozuo Jiulishan Mine & I & 50 & 232 & 2155 \\
Anyang Longshan Mine & I & 40 & 171 & 2340 \\
Hebi 6\# Mine & I & 45 & 223 & 2018 \\
Pingdingshan 6\# Mine & II & 40 & 242 & 1653 \\
Jiaozuo Jiulishan Mine & II & 55 & 350 & 1571 \\
Anyang Longshan Mine & III & 40 & 441 & 907 \\
Hebi 6\# Mine & III & 40 & 415 & 964 \\
Pingdingshan 8\# Mine & IV & 35 & 465 & 753 \\
Jiaozuo Jiulishan Mine & IV & 30 & 472 & 636 \\
\hline
\end{tabular}


From the above experimental data, the ultrasonic velocities in different types of coal structure have the following features:

(1) The ultrasonic velocity varied clearly between types of coal structure.

From type I coal to type IV coal, the ultrasonic velocity gradually slowed down. In type I coal, the ultrasonic velocity was mostly above $2,000 \mathrm{~m} / \mathrm{s}$, and reached $3,300 \mathrm{~m} / \mathrm{s}$ in some samples; In type II coal, the ultrasonic velocity mainly fell in $1,500 \mathrm{~m} / \mathrm{s}$ and $2,000 \mathrm{~m} / \mathrm{s}$; In type III coal, the ultrasonic velocity mostly appeared in $800 \mathrm{~m} / \mathrm{s}-1,000 \mathrm{~m} / \mathrm{s}$; In type IV coal, the ultrasonic velocity primarily remained below $800 \mathrm{~m} / \mathrm{s}$. Furthermore, the ultrasonic velocities in different types of coal structure had overlapping areas. Hence, the adjacent types of coal structure cannot be easily differentiated based on ultrasonic velocity alone.

(2) The ultrasonic velocity varied between the same type of coal samples collected from different sources.

This variation mainly comes from the differences in geological environment. Under different tectonic stresses, the coal samples disagree in density, elastic modulus, Poisson's ratio, as well as rank, which affect their sound properties. That is why coal samples in the same type had different ranges of ultrasonic velocity.

(3) The wave velocities differed significantly between outburst and non-outburst coals.

The ultrasonic velocities of samples in different types from the same mine obeyed the basic law in feature (1): from type I coal to type IV coal, the ultrasonic velocity gradually slowed down. Besides, there was a significant difference between outburst coals (types I and II) and non-outburst coals (types III and IV) in velocity. The ultrasonic velocity in non-outburst coals was at least 2-3 times that in outburst coals.

\subsection{Attenuation coefficient experiments}

The attenuation coefficient is another key parameter for coal medium detection. The value of the coefficient directly reflects how the coal attenuates the ultrasonic signal. Hence, the attenuation coefficient plays an important role in the selection of the working frequency of the ultrasonic probe, and the study on the attenuation law of the ultrasonic signal in coal. This subsection attempts to clarify the relationship between attenuation coefficient and each type of coal structure through experiments, providing a guide for selecting the working frequency of the ultrasonic probe.

As mentioned before, the attenuation coefficient of coal mainly contains two parts: absorption attenuation and diffusion attenuation. Since the two parts are not easy to measure, the mean attenuation coefficient was measured directly in our experiments. Previous research $[17,18]$ shows that the attenuation coefficient of coal can be solved from the amplitude difference $\Delta \mathrm{B}$ and travel difference $\left(x_{2}-x_{1}\right)$ of the received ultrasonic signals: $a=\frac{1}{x_{2}-x_{1}} \cdot \ln \frac{A_{1}}{A_{2}}=\frac{2.3 \Delta B}{20\left(x_{2}-x_{1}\right)}$.

As shown in Figure 4, a set of ultrasonic transmitter and receiver was deployed along the radial direction of the test sample, in addition to the system for ultrasonic velocity experiments. During the experiments, the travel difference of ultrasonic signal was calculated as the difference between the axial length and radial length of the sample; the attenuation coefficient of the sample was calculated by the said formula, based on the measured amplitude difference between the signals received in two directions. Some of the experimental data are recorded in Tables 2-5.

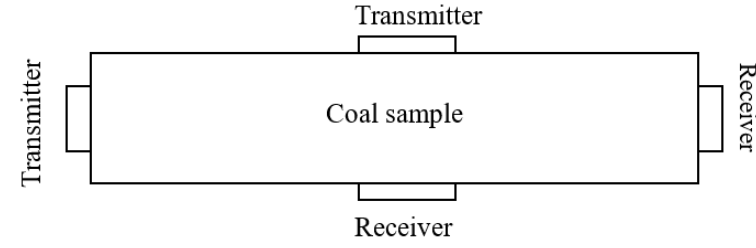

Figure 4. The system structure for attenuation coefficient experiments

Table 2. The attenuation coefficients of different types of coal structures at $20 \mathrm{kHz}$

\begin{tabular}{ccccc}
\hline $\begin{array}{c}\text { Type of } \\
\text { coal } \\
\text { structure }\end{array}$ & $\begin{array}{c}\text { Length-width } \\
\text { difference of } \\
\text { sample } \Delta \mathbf{x}(\mathbf{m})\end{array}$ & $\begin{array}{c}\text { Transverse LongitudinalAttenuation } \\
\text { amplitude } \\
\mathbf{A}_{1}(\mathbf{d B})\end{array}$ & $\begin{array}{c}\text { amplitude } \\
\mathbf{A 2}(\mathbf{d B})\end{array}$ & $\begin{array}{c}\text { coefficient } \\
\boldsymbol{a}\end{array}$ \\
\hline I & 0.2 & 86.3 & 85.8 & 0.251 \\
II & 0.25 & 78.6 & 77.2 & 0.626 \\
III & 0.15 & 66.4 & 60.9 & 4.18 \\
IV & 0.1 & 54.3 & 50.3 & 9.05 \\
\hline
\end{tabular}

Table 3. The attenuation coefficients of different types of coal structures at $25 \mathrm{kHz}$

\begin{tabular}{ccccc}
\hline $\begin{array}{c}\text { Type of } \\
\text { coal } \\
\text { structure }\end{array}$ & $\begin{array}{c}\text { Length-width Transverse Longitudinal } \\
\text { difference of } \\
\text { sample } \mathbf{\Delta x}(\mathbf{m})\end{array}$ & $\begin{array}{c}\text { amplitude } \\
\mathbf{A}(\mathbf{d B})\end{array}$ & $\begin{array}{c}\text { amplitude } \\
\mathbf{A 2}(\mathbf{d B})\end{array}$ & $\begin{array}{c}\text { Attenuation } \\
\text { coefficient } \boldsymbol{a}\end{array}$ \\
\hline I & 0.2 & 79.5 & 78.9 & 0.341 \\
II & 0.25 & 71.3 & 69.5 & 0.783 \\
III & 0.15 & 61.2 & 53.8 & 5.61 \\
IV & 0.05 & 49.6 & 44.6 & 11.45 \\
\hline
\end{tabular}

Table 4. The attenuation coefficients of different types of coal structures at $50 \mathrm{kHz}$

\begin{tabular}{ccccc}
\hline $\begin{array}{c}\text { Type of } \\
\text { coal } \\
\text { structure }\end{array}$ & $\begin{array}{c}\text { Length-width } \\
\text { difference of } \\
\text { sample } \Delta \mathbf{x}(\mathbf{m})\end{array}$ & $\begin{array}{c}\text { Transverse } \\
\text { amplitude } \\
\mathbf{A}_{\mathbf{1}}(\mathbf{d B})\end{array}$ & $\begin{array}{c}\text { Longitudinal } \\
\text { amplitude } \\
\mathbf{A}_{2}(\mathbf{d B})\end{array}$ & $\begin{array}{c}\text { Attenuation } \\
\text { coefficient }\end{array}$ \\
\hline I & 0.2 & 76.2 & 75.0 & 0.681 \\
II & 0.25 & 66.7 & 63.3 & 1.567 \\
III & 0.15 & 52.1 & 37.5 & 11.22 \\
IV & 0.05 & 42.1 & 32.9 & 20.91 \\
\hline
\end{tabular}

Table 5. The attenuation coefficients of different types of coal structures at $100 \mathrm{kHz}$

\begin{tabular}{ccccc}
\hline $\begin{array}{c}\text { Type of } \\
\text { coal } \\
\text { structure }\end{array}$ & $\begin{array}{c}\text { Length-width Transverse Longitudinal } \\
\text { difference of } \\
\text { sample } \Delta \mathbf{x}(\mathbf{m})\end{array}$ & $\begin{array}{c}\text { amplitude } \\
\mathbf{A}(\mathbf{d B})\end{array}$ & $\begin{array}{c}\text { amplitude } \\
\mathbf{A}_{\mathbf{2}}(\mathbf{d B})\end{array}$ & $\begin{array}{c}\text { Attenuation } \\
\text { coefficient } \boldsymbol{a}\end{array}$ \\
\hline I & 0.2 & 71.5 & 69.1 & 1.362 \\
II & 0.25 & 61.7 & 54.9 & 3.134 \\
III & 0.15 & 47.3 & 18.1 & 22.43 \\
IV & 0.05 & 27.5 & 7.8 & 45.2 \\
\hline
\end{tabular}

After analyzing the experimental data, the following conclusions were drawn:

(1) Different types of coal structure had different attenuation coefficients.

In general, the attenuation coefficient increased significantly from type I coal to type IV coal.

(2) Outburst coals differed significantly from non-outburst coals in attenuation coefficient.

The larger the difference in type of coal structure, the greater the variation in attenuation coefficient. According to the experimental data, the attenuation coefficient of type IV coal was more than 100 times that of type I coal. This echoes 
with the conclusions that non-outburst coals are more favorable than outburst coals for the propagation of ultrasonic signal.

(3) There was a close relationship between attenuation coefficient and ultrasonic frequency.

As shown in Figure 5, attenuation coefficient increased with the ultrasonic frequency; the higher the frequency, the larger the increment.

To better reflect the relationship between the working frequency of ultrasonic probe and the attenuation coefficient of coal, the attenuation coefficients of different types of coal structures were fitted as shown in Figure 5.

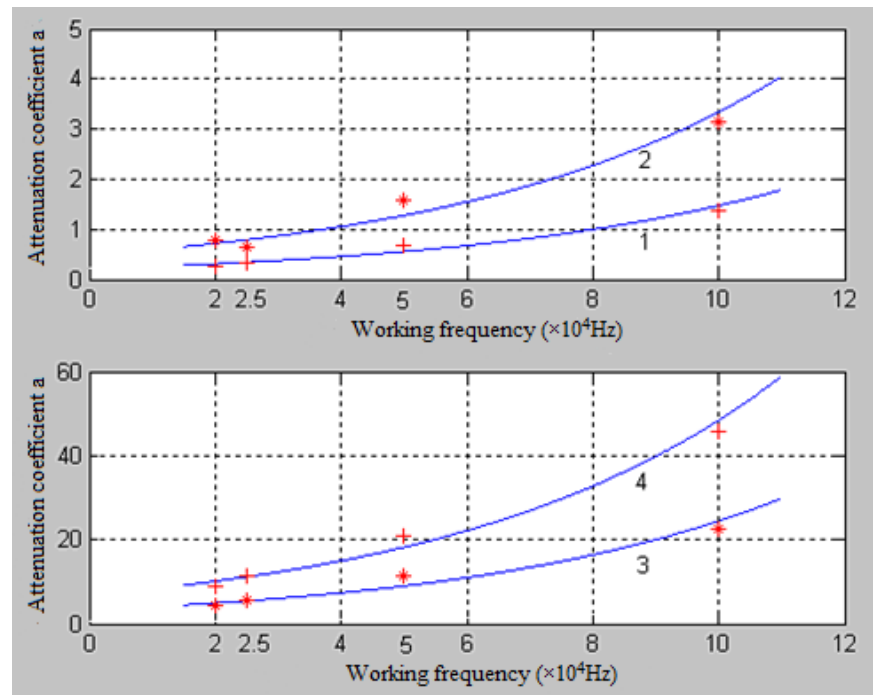

Note: Curves 1-4 are the fitted curves for types I-IV coals, respectively

Figure 5. The fitted curves between attenuation coefficient and working frequency

Two obvious trends could be inferred from the four curves in Figure 5:

(1) In all four types of coal structure, the attenuation coefficient increased at a growing rate with working frequency.

(2) Under the same working frequency, the attenuation coefficient increased as the type of coal structure changed from type I to type IV; the higher the frequency, the larger the difference between the four types of coal structure in attenuation coefficient.

The experimental results confirm the close correlation between the attenuation coefficient and the working frequency of ultrasonic probe: the higher the frequency, the larger the coefficient, and the faster the attenuation of the ultrasonic signal. During the design of detection scheme, the working frequency should be rationalized after comprehensive consideration of the attenuation coefficient, detection purpose, and detection requirements. For our research, the working frequency of ultrasonic probe should be minimized to ensure the detection distance of ultrasonic signal in coal.

\subsection{Model training and verification}

To optimize its parameters, BPNN needs to self-learn lots of experimental data. Here, the massive experimental data are divided into learning samples and verification samples [26, 27]. The learning samples were used for the self-learning and training of the network (Table 6). BPNN relies on gradient descent for self-learning. Before the learning, the input data must be normalized. The learning rate, minimum mean squared error, momentum coefficient, and maximum number of iterations were set to $0.4,0.001,0.8$, and 15,000, respectively. After the learning samples were imported, the network converged after 5,960 iterations.

The trained BPNN was verified with the verification data (Table 7). The verification results show that the network output was basically the same with the actual type of coal structure. This means our model could correctly and reliably identify the type of coal structure.

Table 6. Some samples for BPNN learning and training

\begin{tabular}{|c|c|c|c|c|c|c|}
\hline $\begin{array}{c}\text { Number of } \\
\text { sample group }\end{array}$ & $\begin{array}{c}\mathbf{X}_{1} \\
\mathbf{v}_{\mathrm{p}}(\mathbf{m} / \mathbf{s})\end{array}$ & $\begin{array}{c}\mathrm{X}_{2} \\
a(\mathrm{~dB} / \mathrm{m})\end{array}$ & $\begin{array}{c}\mathbf{X}_{3} \\
f(\mathbf{H z})\end{array}$ & $\mathbf{Y}_{\mathbf{i}}$ & $\mathbf{Y}$ & $\begin{array}{l}\text { Type of coal } \\
\text { structure }\end{array}$ \\
\hline \multirow{3}{*}{ thes of } & 2,340 & 0.251 & 20,000 & 1.01 & 1 & I \\
\hline & 1,896 & 0.626 & 20,000 & 1.77 & 2 & II \\
\hline & 753 & 9.05 & 20,000 & 3.84 & 4 & IV \\
\hline \multirow[b]{2}{*}{2} & 2,130 & 0.295 & 20,000 & 0.97 & 1 & I \\
\hline & 907 & 4.18 & 20,000 & 3.25 & 3 & III \\
\hline \multirow{3}{*}{3} & 2,151 & 0.341 & 25,000 & 1.24 & 1 & I \\
\hline & 1,659 & 0.783 & 25,000 & 2.03 & 2 & II \\
\hline & 1,120 & 5.61 & 25,000 & 3.08 & 3 & III \\
\hline \multirow{3}{*}{4} & 2,236 & 0.681 & 50,000 & 0.94 & 1 & I \\
\hline & 1,578 & 1.567 & 50,000 & 1.93 & 2 & II \\
\hline & 875 & 11.22 & 50,000 & 3.11 & 3 & III \\
\hline \multirow{2}{*}{5} & 1,470 & 1.085 & 25,000 & 2.15 & 2 & II \\
\hline & 647 & 11.45 & 25,000 & 3.94 & 4 & IV \\
\hline \multirow{2}{*}{6} & 970 & 10.87 & 50,000 & 2.91 & 3 & III \\
\hline & 651 & 20.91 & 50,000 & 3.91 & 4 & IV \\
\hline \multirow{2}{*}{7} & 2,018 & 1.362 & 100,000 & 1.26 & 1 & I \\
\hline & 1,723 & 3.134 & 100,000 & 1.80 & 2 & II \\
\hline \multirow{2}{*}{8} & 1,029 & 22.43 & 100,000 & 3.02 & 3 & III \\
\hline & 750 & 33.65 & 100,000 & 4.16 & 4 & IV \\
\hline
\end{tabular}

Note: $Y$ is the actual type of coal structure; $1-4$ are the four types of coal structure, respectively; $Y_{i}$ is the output of the BPNN-based identification model.

Table 7. Some samples for BPNN verification

\begin{tabular}{ccccccc}
\hline $\begin{array}{c}\text { Number of } \\
\text { sample group } \mathbf{v}_{\mathbf{p}}(\mathbf{m} / \mathbf{s}) \boldsymbol{a}(\mathbf{d B} / \mathbf{m})\end{array}$ & $\begin{array}{c}\mathbf{X}_{\mathbf{1}} \\
\mathbf{f}(\mathbf{H z})\end{array}$ & $\mathbf{Y}_{\mathbf{i}}$ & $\mathbf{Y}$ & $\begin{array}{c}\text { Type of coal } \\
\text { structure }\end{array}$ \\
\hline \multirow{2}{*}{1} & $2,152.9$ & 0.2395 & 20,000 & 1.316 & I & I \\
& 887 & 4.09 & 20,000 & 3.18 & 3 & III \\
& 769 & 8.972 & 20,000 & 4.217 & 4 & IV \\
2 & 2,163 & 0.324 & 25,000 & 1.153 & 1 & I \\
& 1,672 & 0.845 & 25,000 & 2.017 & 2 & II \\
& 628 & 10.97 & 25,000 & 4.09 & 4 & IV \\
3 & 1,546 & 1.584 & 50,000 & 2.163 & 2 & II \\
& 1,042 & 10.68 & 50,000 & 2.973 & 3 & III \\
4 & 1,019 & 24.75 & 100,000 & 3.102 & 3 & III \\
& 695 & 41.69 & 100,000 & 3.831 & 4 & IV \\
\hline
\end{tabular}

\section{CONCLUSIONS}

This paper firstly theoretically analyzes the ultrasonic properties of different types of coal structure, and then establishes an intelligent identification model for the type of coal structure, based on the ultrasonic parameters of coal. The following conclusions were drawn from the research:

(1) Different types of coal structure differed significantly in internal structure. The structural differences lead to marked variation in ultrasonic velocity in coal. Besides, the ultrasonic velocities in different types of coal structure partly overlap each other. Thus, it is easy to misjudge the type of coal structure based on ultrasonic velocity alone.

(2) The types of coal structure correspond with the attenuation coefficients of ultrasonic wave. From type I to type 
IV, the attenuation coefficient increased significantly. The greater the structural difference, the more significant the change in the attenuation coefficient.

(3) The attenuation coefficient is closely related to ultrasonic frequency. The higher the frequency, the larger the coefficient, and the faster the ultrasonic signal attenuates. This means the impact of ultrasonic frequency must be considered while applying the attenuation coefficient.

(4) The BPNN-based identification model recognizes the type of coal structure through comprehensive analysis of multiple parameters of the ultrasound passing through the coal: velocity, attenuation coefficient, and frequency, providing an effective way to differentiate between similar types of coal structures. Experimental results show that the proposed model can accurately identify the type of coal structure, laying a solid basis for preventing heat-induced gas outburst and promoting energy-efficient mining.

\section{ACKNOWLEDGMENT}

This project is supported by the Natural Science Foundation of Henan Province, China (Grant No.: 202300410182), National Natural Science Foundation of China (Grant No.: U1704129, 51974109, and 51904095), and the Fundamental Research Funds for the Universities of Henan Province, China (Grant No.: NSFRF180330).

\section{REFERENCES}

[1] Li, M., Jiang, B., Lin, S., Lan, F., Wang, J. (2013). Structural controls on coalbed methane reservoirs in Faer coal mine. Southwest China. Journal of Earth Science, 24(3): 437-448. https://doi.org/10.1007/s12583-0130340-3

[2] Liu, J., Jiang, B., Li, M., Qu, Z., Wang, L., Li, L. (2015). Structural control on pore-fracture characteristics of coals from Xinjing coal mine, northeastern Qinshui basin, China. Arabian Journal of Geosciences, 8(7): 4421-4431. https://doi.org/10.1007/s12517-014-1551-3

[3] Prasad, P.P., Dhanam, K., Kishore, P.P., Mysaiah, D., Seshunarayana, T. (2015). High resolution seismic reflection studies in Godavari coal fields: Mapping of coal seams and associated structural features. Journal of the Geological Society of India, 86(3): 317-323. https://doi.org/10.1007/s12594-015-0317-1

[4] Thong-un, N., Hirata, S., Orino, Y., Kurosawa, M.K. (2015). A linearization-based method of simultaneous position and velocity measurement using ultrasonic waves. Sensors and Actuators A: Physical, 233: 480-499. https://doi.org/10.1016/j.sna.2015.07.029

[5] Kim, M.G., Yoon, S., Kim, H.H., Shung, K.K. (2016). Impedance matching network for high frequency ultrasonic transducer for cellular applications. Ultrasonics, $\quad 65: \quad 258-267$ https://doi.org/10.1016/j.ultras.2015.09.016

[6] Furuno, Y., Tanaka, Y., Nakatoh, Y. (2016). Detecting system of approaching vehicles using an ultrasonic wave. In 2016 IEEE International Conference on Consumer Electronics (ICCE), 393-394. https://doi.org/10.1109/ICCE.2016.7430661

[7] Shumskii, I.P. (1975). Ultrasonic method of estimating the structure and fissuring of coals. Soviet Mining, 11(2):
147-149. https://doi.org/10.1007/BF02501153

[8] Lu, S., He, J. (1997). Ultrasonic characteristics of the disturbed coal mass. Journal of Central South University of Technology, 4(1): 42-45. https://doi.org/10.1007/s11771-997-0028-x

[9] Tang, Z., Zhai, C., Li, Y. (2017). The attenuation of ultrasonic waves in coal: the significance in increasing their propagation distance. Natural Hazards, 89(1): 57-77. https://doi.org/10.1007/s11069-017-2952-x

[10] Matsuo, K., Barolli, L. (2018). A multi positions detection system with supersonic sensors for omnidirectional wheelchair tennis. In International Conference on Broadband and Wireless Computing, Communication and Applications, 25: 637-647. https://doi.org/10.1007/978-3-030-02613-4_57

[11] Mulazzani, L., Salvatore, V., Ravaioli, F., Allegretti, G., Matassoni, F., Granata, R., Piscaglia, F. (2018). Correction to: Point shear wave ultrasound elastography with Esaote compared to real-time 2D shear wave elastography with supersonic imagine for the quantification of liver stiffness. Journal of ultrasound, 21(2): 169. https://doi.org/10.1007/s40477-018-0293-6

[12] Wu, M., Yue, H., Cao, M., Hou, X., Yu, H. (2018). Research on forebody active disturbed flow characteristics of slender body in supersonic field. Journal of the Brazilian Society of Mechanical Sciences and Engineering, 40(9): 431. https://doi.org/10.1007/s40430-018-1325-z

[13] Xu, Y., Wang, Q., Jiang, X., Zu, H., Wang, W., Feng, R. (2020). Nondestructive assessment of microcracks detection in cementitious materials based on nonlinear ultrasonic modulation technique. Construction and Building Materials, 267: 121653. https://doi.org/10.1016/j.conbuildmat.2020.121653

[14] Zhao, M., Nie, Z., Wang, K., Liu, P., Zhang, X. (2019). Nonlinear ultrasonic test of concrete cubes with induced crack. Ultrasonics, 97: 1-10. https://doi.org/10.1016/j.ultras.2019.04.002

[15] Li, W., Frederick, H., Palardy, G. (2020). Multifunctional films for thermoplastic composite joints: Ultrasonic welding and damage detection under tension loading. Composites Part A: Applied Science and Manufacturing, 141: 106221. https://doi.org/10.1016/j.compositesa.2020.106221

[16] Ji, B., Zhang, Q., Cao, J., Li, H., Zhang, B. (2021). Noncontact detection of delamination in stainless steel/carbon steel composites with laser ultrasonic. Optik, 226 :

165893. https://doi.org/10.1016/j.ijleo.2020.165893

[17] Ben, B.S., Ben, B.A., Vikram, K.A., Yang, S.H. (2013). Damage identification in composite materials using ultrasonic based Lamb wave method. Measurement, 46(2): 904-912. https://doi.org/10.1016/j.measurement.2012.10.011

[18] Xue, M., Su, M., Cai, X. (2007). Measuring particle size and concentration of dense slurry by ultrasonic method. In Challenges of Power Engineering and Environment, 652-655. https://doi.org/10.1007/978-3-540-76694$0 \quad 122$

[19] Wang, J., Wang, C., Han, Z. (2020). Multi-frequency ultrasonic underground cavity scanning detection technology in borehole. Measurement, 161: 107889. https://doi.org/10.1016/j.measurement.2020.107889

[20] Song, S., Miao, Z. (2015). Research on vehicle type 
classification based on spatial pyramid representation and bp neural network. In International Conference on Image and Graphics, 9219: 188-196. https://doi.org/10.1007/978-3-319-21969-1_17

[21] He, F., Zhang, L. (2018). Mold breakout prediction in slab continuous casting based on combined method of GA-BP neural network and logic rules. The International Journal of Advanced Manufacturing Technology, 95(912): 4081-4089. https://doi.org/10.1007/s00170-0171517-1

[22] Duan, Y., Yuan, L.L. (2015). Risk assessment of aviation maintenance error based on set pair analysis and bp neural network. In Proceedings of the 5th International Asia Conference on Industrial Engineering and Management Innovation (IEMI2014), 1: 299-303. https://doi.org/10.2991/978-94-6239-100-0 55

[23] Chang, K.C., Zhou, Y.W., Wang, H.C., Lin, Y.C., Chu, K.C., Hsu, T.L., Pan, J.S. (2020). Study of PSO Optimized BP neural network and smith predictor for MOCVD temperature control in $7 \mathrm{~nm} 5 \mathrm{G}$ chip process. In International Conference on Advanced Intelligent Systems and Informatics, 1261: 568-576. https://doi.org/10.1007/978-3-030-58669-0_51
[24] Mao, C., Yang, Z., Gao, C. (2018). Prediction of auricularias bacterial package density based on improved bp neural network. In International Conference on Applications and Techniques in Cyber Security and Intelligence, $\quad 842$ : https://doi.org/10.1007/978-3-319-98776-7_136

[25] Li, Y., Wan, Y., Xiao, J., Zhu, Y. (2019). Prediction of photovoltaic power generation based on POS-BP neural network. In International Conference on Bio-Inspired Computing: Theories and Applications, 1160: 443-453. https://doi.org/10.1007/978-981-15-3415-7_37

[26] Zhang, R., Liu, M., Zhang, Q., Cai, Z. (2020). A network security situation prediction algorithm based on BP neural network optimized by SOA. In International Conference on Artificial Intelligence and Security, 12240 : 417-427. https://doi.org/10.1007/978-3-030-57881-7_37

[27] Dong, G., Li, W., Wang, S., Zhang, X., Lu, J., Li, X. (2018). The assessment method of network security situation based on improved BP neural network. In International Conference on Computer Engineering and Networks, 905: 67-76. https://doi.org/10.1007/978-3030-14680-1_9 